\title{
Polymorphism at the tetranucleotide repeat locus DYS389 in 10 populations reveals strong geographic clustering
}

\author{
B urkhard R olf ${ }^{1}$, E ckhard M eyer ${ }^{2}$, B ernd Brinkmann ${ }^{1}$ and Peter de K nijff ${ }^{3}$ \\ ${ }^{1}$ Institut für R echtsmedizin, Westfälische-Wilhelms Universität, M ünster, G ermany \\ II nstitut für R echtsmedizin, Friedrich-Schiller-U niversität, J ena, G ermany \\ ${ }^{3} \mathrm{~L}$ eiden University M edical Center, L eiden, The Netherlands
}

\begin{abstract}
Several short tandem repeat polymorphism loci at the non-recombining part of the $Y$ chromosome have been described recently and are now widely used for the investigation of the history and the diversity of man. ${ }^{1-5}$ The tetranucleotide repeat polymorphism at the DYS389 locus consists of two repetitive stretches with different numbers of $(T C T G)_{n}(T C T A)_{m}$ repeat units. To study the overall variability of this locus, 768 alleles from males from 10 human populations (two sub-Saharan African, four Caucasoid and four Asian/Amerind populations) were investigated. The alleles found in the populations of different geographic origin exhibited remarkable differences in the number and arrangement of repeats in the two repetitive stretches and up to nine different sequence variants for a single fragment length have been detected. So far $\mathbf{5 3}$ different alleles, ie haplotypes, have been observed. Analysis of molecular variance (AMOVA) indicates that at least $24.5 \%$ of the total genetic variance was found between the populations and that these differences were significant in most pairwise comparisons. We propose a model, in which both founder effects and genetic drift together with single step replication slippage mutations explain the picture of haplotype diversity observed with this single locus.
\end{abstract}

Keywords: tetranucleotide; DYS389; males; network; y chromosome

\section{Introduction}

A utosomal short tandem repeat (STR) systems are used in human identification/parentage ${ }^{6}$ linkage analy$\mathrm{sis}^{7}{ }^{7}$ and for the investigation of human evolution. ${ }^{8}$ R ecently, microsatellites at the male-specific part of the

Correspondence: Prof. Dr. B. Brinkmann, Institut für R echtsmedizin, Westfälische-Wilhelms U niversität, von E smarch Straße 62, D -48129 M ünster, G ermany. Tel.: 49251 8355160; Fax: 492518355158

R eceived 10 D ecember 1997; revised 23 M arch 1998; accepted 15 A pril 1998
$Y$ chromosome have been described and are now increasingly used for the study of male-specific lineage evolution. ${ }^{4,5,9}$ In contrast to autosomal microsatellites, variations observed in several loci can be combined and studied via haplotype analysis, because no recombination occurs (as long as one only considers variation outside the pseudo autosomal region). A s there is only one $Y$ chromosome to every four autosomes, genetic variations on the $Y$ chromosome are much more prone to effects of drift. This is also true of $Y$ haplotypes in relation to mtDNA haplotypes, as the reproduction success of males can differ strongly, whereas the 
number of offspring of females is probably less variable. Paternal inheritance allows investigation of male lineages over many generations. ${ }^{10}$

The tetranucleotide repeat polymorphism DYS389 ${ }^{11}$ consists of two stretches with different numbers of $(\mathrm{TCTG})_{\mathrm{n}}(\mathrm{TCTA})_{\mathrm{m}}$ repeat units, separated by 48 base pairs. To study the variability of this locus, 768 alleles from males from 10 populations were investigated either by sequencing or by a nested PCR approach.

\section{Materials and Methods}

\section{Population Samples}

The population samples were Ovambos (Bantu) from Namibia, Pygmies from Central A frica, Caucasoid G ermans from northwest $\mathrm{G}$ ermany, D utch males from all over $\mathrm{H}$ olland, $M$ oroccans living in B russels, B elgium, Turks from the $A$ dana area, Chinese ( $\mathrm{H}$ an race) from the Shenyang area, Japanese from the Shiga area, Inuit from southwest Greenland, and Surinamese from either Surinam or living in Holland.

\section{Determination of Haplotypes}

DNA was isolated from dried blood on cotton according to standard procedures ${ }^{12}$ and quantified using the slot blot technique. ${ }^{13}$ Fragment length determination was carried out on an A BI-373-D NA -Sequencer (primer R 1 FA M and R 4N HEX labelled) or an ALF-system (Pharmacia, U ppsala, Sweden, primer F2 FITC-labelled). The PCR-primer sequences were:

\section{F 1: CCA ACTCTCATCTGTATTATCTAT \\ F2: TCTGTATTATCTATGTGTG \\ R 1: TCTTATCTCCACCCACCAGA \\ R2: CCAGACATTGCCAAGTGTTACTTG \\ R 3: ATACAGAAGTAGGTATAA \\ R 4N : ATA A ATA ATATA A A ATATA A ATA A ATA A A TATAGATAGACA G}

A mplification was carried out with $2 \mathrm{ng}$ of template D NA, 1 unit Taq-D NA-polymerase, $200 \mu \mathrm{m}$ of each dNTP, $2 \mu \mathrm{l}$ $10 \times P C R$ reaction buffer containing $500 \mathrm{~mm} \mathrm{KCl}, 100 \mathrm{~mm}$ Tris/ $\mathrm{HCl}, 15 \mathrm{~mm} \mathrm{M} \mathrm{gCl} 1_{2}, 1 \%$ Triton $\mathrm{X}-100, \mathrm{pH} 8.8$ at $25^{\circ} \mathrm{C}$, $250 \mu \mathrm{g} \mathrm{BSA}$ and $1 \mu \mathrm{m}$ of each primer in a final volume of $25 \mu \mathrm{l}$. A mplification conditions were $94^{\circ} \mathrm{C}$ for 1 min followed by 30 cycles of $94^{\circ} \mathrm{C}$ for $45 \mathrm{~s}, 55^{\circ} \mathrm{C}$ for $20 \mathrm{~s}$ and $72^{\circ} \mathrm{C}$ for $30 \mathrm{~s}$.

A lleles from males were sub-typed either by sequencing or by a nested PCR approach. Sequencing was carried out in both directions with the product of the PCR 3 reaction on an A BI-373-D NA -Sequencer using the Taq D ye-D eoxy-Terminator Cycle Sequencing Kit (Perkin Elmer/A pplied Biosystems, Foster City, CA, USA ). E very sequence was determined in both directions with primer $F 2$ and $R 2$, respectively. For the semi-nested PCR approach, $1 \mu \mathrm{L}$ of a 1 to 100 dilution of the product of PCR 1 (Figure 1 ) was used as template in a semi-nested reaction PCR 2 (Figure 1 ) using the same PCR conditions. The fragment length of the resulting four fragments was determined on the A BI-system. The haplotypes were obtained by the subtraction of the fragment lengths of

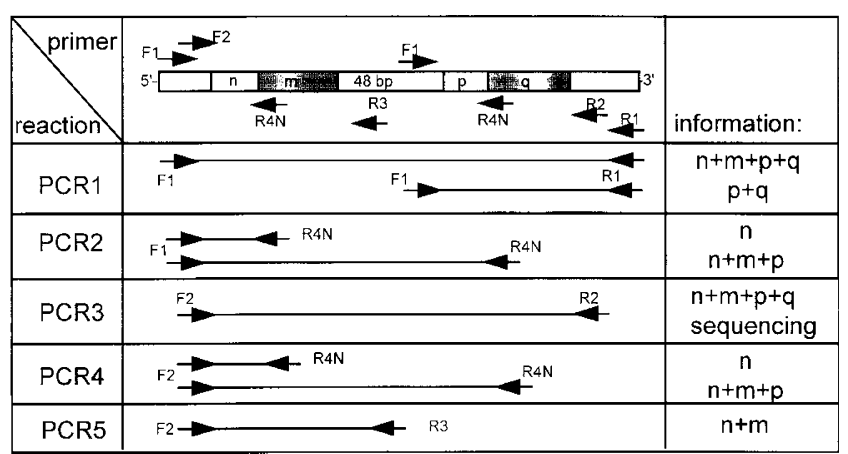

Figure 1 Sequence structure, primer binding sites and $P C R$ products generated from locus DY S389. For primer sequences and PCR conditions see M aterials and $M$ ethods.

the four different products. A Iternatively, $1 \mu \mathrm{L}$ of a 1 to 100 dilution of the product of PCR 3 (Figure 1) was used as a template in the semi-nested reaction PCR 4 (Figure 1). The fragment length of the resulting fragments was determined on the A LF-system. Together with the information from PCR 5, the haplotypes could again be obtained by subtraction of the fragment lengths of the four different products. B oth methods were calibrated with 30 sequenced alleles.

\section{Results}

The initially developed set of primers for this locus [G enome D atabase, ID : G 00-366-108] yielded two PCR fragments because one primer had two annealing sites. ${ }^{1}$ To allow for direct sequencing and further sub-typing of the PCR -products, we redesigned the primers in order to obtain only one product. Fragment length determination of the resulting single PCR product of males from two sub-Saharan A frican populations, four Caucasoid populations and four A sian/A merind populations gave 9 length variants from 288 to $320 \mathrm{bp}$.

The structure of all alleles is (TCTG) $4-7$ (TCTA ) ${ }_{10-14}(48 \mathrm{bp})(\text { TCTG })_{3}(\text { TCTA })_{8-13}$ and is possibly the result of duplication of one initial repeat motif; 53 different alleles, ie haplotypes, were observed (Table 1). The differences arise from the number and arrangement of repeats in the two repetitive stretches (Table 1). Up to nine different variants for single fragment length have been detected. The allele described by Cooper et al ${ }^{1}$ (1996, E M BL accession N o. 97312) consisting of (TCTG $)_{5}(\text { TCTA })_{12}(48 \mathrm{bp})$ $(T C T G)_{3}(T C T A)_{10}$ was found 58 times in seven of our 10 population samples (Table 1), haplotype \#25); additionally we found eight other haplotypes of the same length but with different arrangement of the repeats. The most frequent haplotype in our study was $(\text { TCTG })_{5}(\text { TCTA })_{11}(48 \mathrm{bp})(\text { TCTG })_{3}(\text { TCTA })_{10}$. It was 
Table 1 R epeat structure and distribution in the populations of the observed 53 haplotyes

\begin{tabular}{|c|c|c|c|c|c|c|c|c|c|c|c|c|c|c|c|}
\hline$\overline{\mathrm{No}}$ & $n+m+q$ & $\mathrm{n}$ & $m$ & $q$ & Dut & Sur & Pyg & Inu & $\mathrm{Mar}$ & Tur & Ger & Jap & Chi & Ova & All \\
\hline 1 & 23 & 4 & 10 & 9 & 0 & 1 & 0 & 0 & 0 & 0 & 0 & 0 & 0 & 0 & 1 \\
\hline 2 & 24 & 4 & 11 & 9 & 0 & 0 & 0 & 0 & 0 & 0 & 0 & 0 & 4 & 0 & 4 \\
\hline 3 & 25 & 4 & 11 & 10 & 0 & 0 & 0 & 0 & 0 & 1 & 0 & 1 & 3 & 0 & 5 \\
\hline 4 & 26 & 4 & 11 & 11 & 0 & 0 & 0 & 0 & 0 & 2 & 0 & 3 & 0 & 0 & 5 \\
\hline 5 & 24 & 4 & 12 & 8 & 0 & 0 & 0 & 0 & 0 & 0 & 0 & 2 & 1 & 0 & 3 \\
\hline 6 & 25 & 4 & 12 & 9 & 0 & 1 & 0 & 0 & 0 & 0 & 0 & 2 & 6 & 0 & 9 \\
\hline 7 & 26 & 4 & 12 & 10 & 0 & 1 & 0 & 0 & 0 & 0 & 1 & 3 & 8 & 0 & 13 \\
\hline 8 & 27 & 4 & 12 & 11 & 0 & 2 & 0 & 0 & 0 & 0 & 4 & 5 & 5 & 0 & 16 \\
\hline 9 & 28 & 4 & 12 & 12 & 0 & 0 & 0 & 0 & 0 & 0 & 0 & 1 & 0 & 0 & 1 \\
\hline 10 & 26 & 4 & 13 & 9 & 0 & 2 & 0 & 0 & 0 & 1 & 0 & 5 & 9 & 0 & 17 \\
\hline 11 & 27 & 4 & 13 & 10 & 0 & 3 & 0 & 0 & 0 & 3 & 0 & 2 & 3 & 0 & 11 \\
\hline 12 & 28 & 4 & 13 & 11 & 0 & 1 & 0 & 0 & 0 & 2 & 0 & 1 & 1 & 0 & 5 \\
\hline 13 & 27 & 4 & 14 & 9 & 1 & 0 & 0 & 0 & 0 & 0 & 0 & 2 & 5 & 0 & 8 \\
\hline 14 & 28 & 4 & 14 & 10 & 0 & 0 & 0 & 0 & 0 & 0 & 0 & 0 & 1 & 0 & 1 \\
\hline 15 & 24 & 5 & 9 & 10 & 1 & 0 & 0 & 0 & 0 & 0 & 1 & 0 & 0 & 0 & 2 \\
\hline 16 & 24 & 5 & 10 & 9 & 0 & 1 & 0 & 0 & 0 & 1 & 2 & 0 & 1 & 0 & 5 \\
\hline 17 & 25 & 5 & 10 & 10 & 9 & 1 & 0 & 0 & 0 & 4 & 5 & 0 & 0 & 0 & 19 \\
\hline 18 & 26 & 5 & 10 & 11 & 1 & 1 & 0 & 0 & 0 & 1 & 2 & 1 & 0 & 0 & 6 \\
\hline 19 & 24 & 5 & 11 & 8 & 0 & 0 & 1 & 0 & 0 & 1 & 1 & 0 & 0 & 0 & 3 \\
\hline 20 & 25 & 5 & 11 & 9 & 18 & 0 & 6 & 4 & 1 & 9 & 26 & 1 & 0 & 0 & 65 \\
\hline 21 & 26 & 5 & 11 & 10 & 31 & 8 & 3 & 8 & 4 & 23 & 81 & 2 & 10 & 0 & 170 \\
\hline 22 & 27 & 5 & 11 & 11 & 8 & 3 & 0 & 20 & 2 & 12 & 10 & 5 & 1 & 0 & 61 \\
\hline 23 & 25 & 5 & 12 & 8 & 0 & 0 & 3 & 0 & 0 & 0 & 0 & 0 & 0 & 0 & 3 \\
\hline 24 & 26 & 5 & 12 & 9 & 3 & 1 & 0 & 1 & 5 & 7 & 10 & 0 & 0 & 1 & 28 \\
\hline 25 & 27 & 5 & 12 & 10 & 9 & 2 & 0 & 1 & 0 & 11 & 31 & 2 & 2 & 0 & 58 \\
\hline 26 & 28 & 5 & 12 & 11 & 2 & 4 & 0 & 14 & 0 & 5 & 5 & 8 & 1 & 0 & 39 \\
\hline 27 & 29 & 5 & 12 & 12 & 0 & 1 & 0 & 9 & 0 & 0 & 1 & 2 & 0 & 0 & 13 \\
\hline 28 & 30 & 5 & 12 & 13 & 0 & 0 & 0 & 1 & 0 & 0 & 0 & 0 & 0 & 0 & 1 \\
\hline 29 & 27 & 5 & 13 & 9 & 2 & 0 & 1 & 0 & 1 & 1 & 2 & 0 & 1 & 0 & 8 \\
\hline 30 & 28 & 5 & 13 & 10 & 1 & 0 & 0 & 1 & 1 & 11 & 13 & 1 & 1 & 0 & 29 \\
\hline 31 & 29 & 5 & 13 & 11 & 0 & 0 & 0 & 3 & 0 & 2 & 1 & 2 & 0 & 0 & 8 \\
\hline 32 & 29 & 5 & 14 & 10 & 0 & 1 & 0 & 0 & 0 & 1 & 1 & 0 & 0 & 0 & 3 \\
\hline 33 & 30 & 5 & 14 & 11 & 0 & 0 & 0 & 0 & 0 & 1 & 1 & 0 & 0 & 0 & 2 \\
\hline 34 & 31 & 5 & 15 & 11 & 0 & 0 & 0 & 0 & 0 & 1 & 0 & 0 & 0 & 0 & 1 \\
\hline 35 & 24 & 6 & 9 & 9 & 0 & 0 & 0 & 0 & 0 & 0 & 0 & 0 & 0 & 1 & 1 \\
\hline 36 & 25 & 6 & 9 & 10 & 0 & 0 & 0 & 0 & 0 & 0 & 1 & 0 & 0 & 0 & 1 \\
\hline 37 & 25 & 6 & 10 & 9 & 0 & 1 & 2 & 0 & 0 & 0 & 0 & 0 & 0 & 2 & 5 \\
\hline 38 & 26 & 6 & 10 & 10 & 1 & 0 & 3 & 0 & 6 & 0 & 0 & 0 & 0 & 6 & 16 \\
\hline 39 & 27 & 6 & 10 & 11 & 0 & 0 & 0 & 0 & 16 & 0 & 1 & 0 & 0 & 0 & 17 \\
\hline 40 & 28 & 6 & 10 & 12 & 0 & 0 & 0 & 0 & 2 & 0 & 0 & 0 & 0 & 0 & 2 \\
\hline 41 & 29 & 6 & 10 & 13 & 0 & 0 & 0 & 0 & 1 & 0 & 0 & 0 & 0 & 0 & 1 \\
\hline 42 & 26 & 6 & 11 & 9 & 0 & 1 & 1 & 0 & 0 & 2 & 2 & 0 & 0 & 1 & 7 \\
\hline 43 & 27 & 6 & 11 & 10 & 2 & 9 & 4 & 0 & 2 & 4 & 2 & 0 & 0 & 20 & 43 \\
\hline 44 & 28 & 6 & 11 & 11 & 0 & 2 & 0 & 0 & 1 & 3 & 1 & 0 & 0 & 1 & 8 \\
\hline 45 & 27 & 6 & 12 & 9 & 0 & 2 & 0 & 0 & 0 & 2 & 2 & 0 & 0 & 3 & 9 \\
\hline 46 & 28 & 6 & 12 & 10 & 0 & 4 & 2 & 0 & 1 & 1 & 0 & 0 & 0 & 8 & 16 \\
\hline 47 & 29 & 6 & 12 & 11 & 0 & 2 & 0 & 0 & 0 & 0 & 2 & 0 & 0 & 2 & 6 \\
\hline 48 & 28 & 6 & 13 & 9 & 0 & 0 & 0 & 0 & 1 & 0 & 1 & 0 & 0 & 0 & 2 \\
\hline 49 & 29 & 6 & 13 & 10 & 0 & 0 & 1 & 0 & 0 & 1 & 0 & 0 & 0 & 2 & 4 \\
\hline 50 & 29 & 7 & 11 & 11 & 0 & 0 & 1 & 0 & 0 & 0 & 0 & 0 & 0 & 1 & 2 \\
\hline 51 & 30 & 7 & 11 & 12 & 0 & 0 & 2 & 0 & 0 & 0 & 0 & 0 & 0 & 0 & 2 \\
\hline 52 & 28 & 7 & 12 & 9 & 0 & 0 & 0 & 0 & 0 & 1 & 0 & 0 & 0 & 0 & 1 \\
\hline 53 & 29 & 7 & 12 & 10 & 0 & 0 & 0 & 0 & 0 & 1 & 0 & 0 & 0 & 1 & 2 \\
\hline SU M & & & & & 89 & 55 & 30 & 62 & 44 & 115 & 210 & 51 & 63 & 49 & 768 \\
\hline
\end{tabular}


found 170 times and was present in nine of our 10 population samples. 0 ther haplotypes were unique in our study and seen only once. To investigate relations between the haplotypes observed, a network was constructed. ${ }^{1,2,14} \mathrm{~A}$ ssuming a single step mutation model, ${ }^{15,16}$ haplotypes differing by only one repeat are called adjacent and connected by a line. Due to the three variable parameters, the resulting network is a three-dimensional figure. $\mathrm{H}$ aplotypes sharing the number of repeats in one motif type, eg four in the most 5 '-repetitive region, are placed in a plane. All 53 haplotypes observed could be connected in this way.
Seven haplotypes are connected with six others, the maximum number of connections that could be achieved in this model. Four haplotypes are only connected once (Figure 2).

Figure 2 highlights the individual haplotypes found in the respective populations. A lmost all populationspecific haplotypes can be connected via the single step lines; however these population-specific nets do not overlap to a great extent. The A sian/I nuit haplotypes all start with four or five TCTG repeats in the most 5'-repeat cases. The haplotypes of Caucasoid males (D utch, G ermans, Turks and M oroccans) all start with
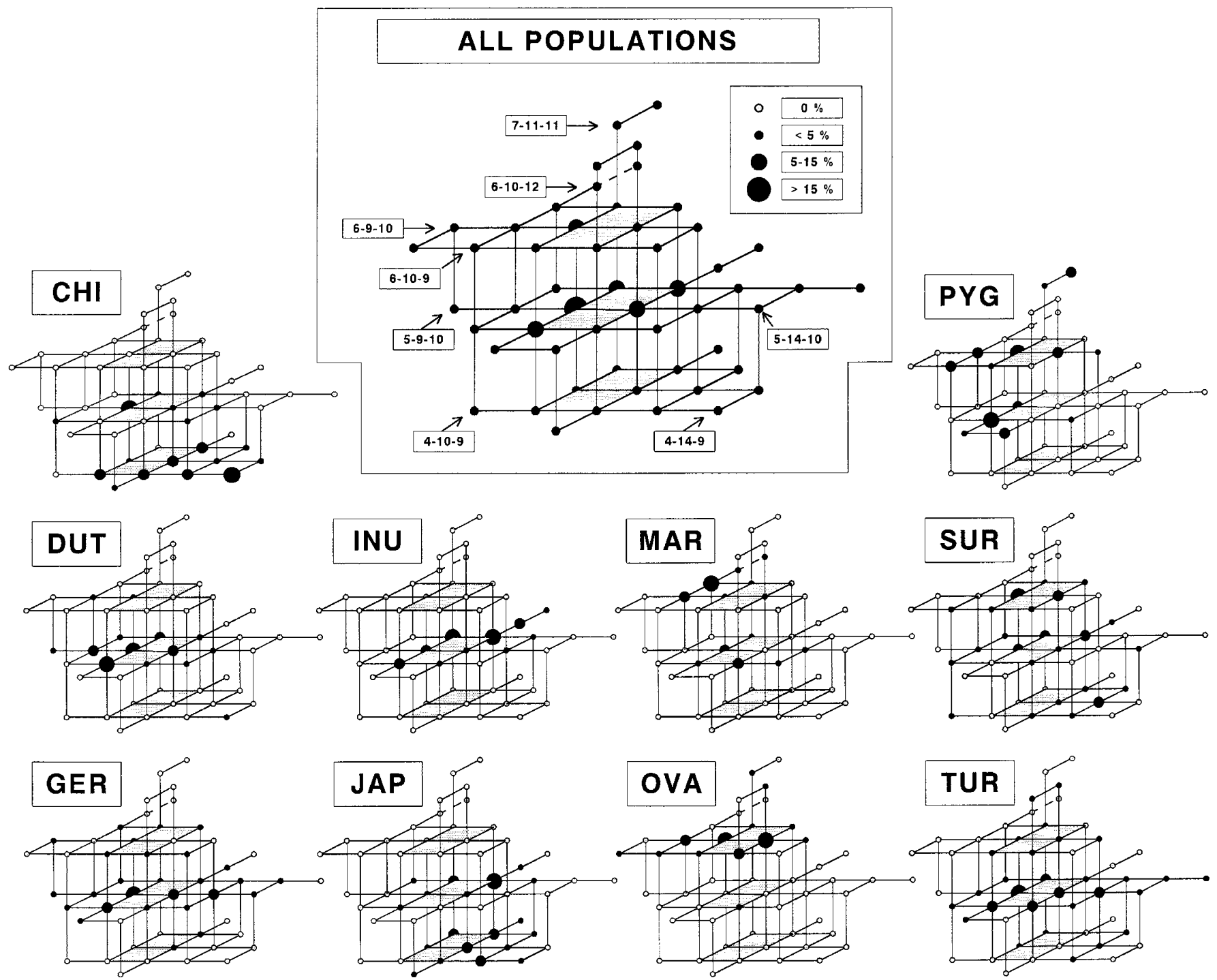

Figure 2 Minimum spanning network of haplotypes of DYS389 locus. All 53 haplotypes observed in this study are shown. $\mathrm{H}$ aplotypes differing by only one repeat are called adjacent and connected by a line. The resulting networks indicate possible transitions caused by single step mutations. E very dimension in the figure indicates length changes in one of the three regions. F requency of the haplotypes is indicated by the size of the nodes. 
Table 2 PhiST-distances between pairs of populations (below diagonal). A bove diagonal is the probability for the statistical significance of the observed distance, derived from 10000 iterations

\begin{tabular}{|c|c|c|c|c|c|c|c|c|c|c|}
\hline$\overline{1}$ & D ut & Sur & Pyg & Inu & Mor & Tur & Ger & Jap & Chi & O va \\
\hline Dut & & 0.0005 & 0.0002 & 0.0000 & 0.0000 & 0.0000 & 0.0077 & 0.0000 & 0.0000 & 0.0000 \\
\hline Sur & 0.0869 & & 0.0000 & 0.0000 & 0.0001 & 0.5293 & 0.0345 & 0.0000 & 0.0000 & 0.0000 \\
\hline Pyg & 0.1385 & 0.1327 & & 0.0000 & 0.0018 & 0.0000 & 0.0000 & 0.0000 & 0.0000 & 0.0188 \\
\hline Inu & 0.3650 & 0.1517 & 0.4382 & & 0.0000 & 0.0000 & 0.0000 & 0.0000 & 0.0000 & 0.0000 \\
\hline Mor & 0.2485 & 0.2006 & 0.1626 & 0.3133 & & 0.0000 & 0.0000 & 0.0000 & 0.0000 & 0.0002 \\
\hline Tur & 0.0947 & -0.0041 & 0.1996 & 0.1852 & 0.2771 & & 0.0089 & 0.6040 & 0.0000 & 0.0000 \\
\hline Ger & 0.0300 & 0.0251 & 0.1742 & 0.2938 & 0.3104 & -0.0231 & & 0.0000 & 0.0000 & 0.0000 \\
\hline Jap & 0.3318 & 0.1563 & 0.3976 & 0.1983 & 0.4430 & 0.1344 & 0.2576 & & 0.0002 & 0.0000 \\
\hline Chi & 0.3807 & 0.2681 & 0.4317 & 0.4419 & 0.5509 & 0.2181 & 0.3188 & 0.1020 & & 0.0000 \\
\hline Ova & 0.3309 & 0.2208 & 0.0669 & 0.4925 & 0.1512 & 0.2689 & 0.3194 & 0.5206 & 0.5867 & \\
\hline
\end{tabular}

four one (one only), five and six TCTG-repeats, whereas the sub-Saharan A frican samples start with five, six or seven (Table 1)

\section{Discussion}

To explain the variability and population specificity observed with this locus, we assume the presence of alleles with four, five, six and seven TCT G -repeats in an ancestral population. Migration processes have spread males of this ancestral population around the world. Founder effects and genetic drift will probably have reduced the initial diversity (eg chromosome number) and thus the variation of the number of TCTG -repeats in modern populations. The haplotype diversity observed within populations today is caused mainly by the number of repeats in both (TCTA) $n$ motifs of the locus. Therefore new mutations are more likely to occur in the longer TCTA strand than in the shorter 5'-TCT G strand. Interestingly, the autosomal tetranucleotide repeat in the intron 31 of the von Willbrandt Factor gene (VWA) has a similar repeat structure of $(T C T G)_{4}(T C T A)_{8-17}$. Variation in length of this STR is almost exclusively due to variations in the number of TCTA repeats, indicating the higher mutability of this repeat motif. The fact that the frequent haplotypes are observed in the centre of the network further supports the single step mutation model, as does the study by $\mathrm{H}$ eyer et al. ${ }^{17}$ who investigated $Y$ chromosomal STR $\mathrm{s}$ in deep rooted pedigrees in order to estimate mutation rates. A II mutations observed in this study were singlestep mutations; the mutation rate was as high as $0.21 \%$ per generation. Therefore the haplotype diversity and the geographic clustering observed is a result of both founder effects and genetic drift together with a high rate of (single-step) mutations.

In order to compare differences in haplotype distribution and the amount of genetic variation included in the $Y$ haplotypes we followed the suggestions of Excoffier et $\mathrm{al}^{18,19}$ and Michalakis and Excoffier ${ }^{20}$ and performed an 'analysis of molecular variance', (A M OVA ) as previously explained in detail by us. ${ }^{3}$ The WINAMOVA and ARLEQUIN programs were used for these analyses. Surprisingly, $24.51 \%$ of the variance was inter-population and the remaining $76.49 \%$ intrapopulation difference. Bootstrapping of the PhiST distance between the populations revealed that 38 of the 45 comparisons were statistically significant (Table 2).

Interestingly, others using network approaches for the investigation of $Y$ chromosomal haplotypes ${ }^{1,2}$ did not observe strong geographic clusters. These authors assumed that high rates of new mutations randomised the haplotypic structures. The data presented in this work allow us to define population-specific haplotypes, because sequencing revealed that part of the locus is highly conserved. Thus, locus DYS389 will become a useful tool for the investigation of human evolution.

\section{References}

1 Cooper G, A mos W, Hoffman D, Rubinsztein DC: Network analysis of human $Y$ microsatellite haplotypes. H um Mol G enet 1996; 5: 1759-1766.

2 D eka R, J in L, Shriver MD, Y U LM et al: Dispersion of human in chromosome haplotypes based on five microsatellites $Y$ global populations. Genome Res 1996; 6: 1177-1184.

3 Roewer $L, K$ ayser $M$, Dieltjes $P$ et al: A nalysis of molecular variance (A M OVA) of $Y$ chromosome specific microsatellites in two closely related human populations. $\mathrm{H}$ um Mol G enet 1996; 5: 1029-1033.

4 Lineares A R, Nayer K, Goldstein D B et al: Geographic clustering of human $Y$ chromosome haplotypes. A nn $\mathrm{H}$ um G enet 1996; 60: 401-408.

5 Sajantila A, Salem A - H, Savolainen P, B auer K, G ierig C, Pääbo $S$ : Paternal and maternal DNA lineages reveal a bottleneck in the founding of the Finnish population. P roc Natl A cad Sci USA 1996; 93: 12035-12039. 
6 Gill P, I vanov PL, K impton C et al: Identification of the remains of the Romanov family by DNA analysis. Nat G enet 1994; 6: 130-135.

7 H earn CM, G hosh S, Todd JA : M icrosatellites for linkage analysis of genetic traits. Trends $G$ enet 1992; 8: 288-294.

8 Bowcock A M, R uiz-Linares A, Tomfohrde J, Minch E, $K$ idd JR, Cavalli-Sforza LL: High resolution of human evolutionary trees with polymorphic microsatellites. Nature 1994; 368: 455-457.

9 Mitchell JR, Hammer M F : H uman evolution and the $Y$ chromosome. Curr O pin G enet Dev 1996; 6: 737-742.

10 Jobling $M A$, Tyler-Smith $C$ Fathers and sons: the $Y$ chromosome and human evolution. Trends $\mathrm{G}$ enet 1995; 11: 449-456.

11 Murray J C: Genome DataB ase: 3661081993

12 Wiegand $\mathrm{P}$, Budowle B, R and, S, B rinkmann B: Forensic validation of the STR systems SE 33 and TC 11. Int J L egal M ed 1993; 105: 315-320.

13 Waye J S, Presley LA, Budowle B, Shutler GS, Fourney RM: A simple and sensitive method for quantifying human genomic DNA in forensic specimen extracts. Biotechniques 1989; 7: 852-855.

14 B andelt HJ, Forster P, Sykes BC, R ichards M B : M itochondrial portraits of human populations using median networks. G enetics 1995; 141: 743-753.
15 Weber $\mathrm{JL}$, Wong C: M utation of human short tandem repeats. Hum M ol G enet, 1993; 2: 1123-1128.

16 Valdes A M, Slatkin M, Freimer N B : A llele frequencies at microsatellite loci: the stepwise mutation model revisited. Genetics 1994; 133: 737-749.

17 Heyer E, Puymirat J, Dieltjes P, Bakker E, de K nijff P: Estimating $Y$ chromosome specific microsatellite mutation frequencies using deep rooting pedigrees: H um M ol G enet 1997; 6: 799-803.

18 Excoffier L, Smouse PE: U sing allele frequencies and geographic subdivision to reconstruct gene trees within a species: Molecular Variance Parsimony. Genetics, 1994; 136: 343-359.

19 Michalakis Y, Excoffier LA : Generic estimation of population subdivision using distances between alleles with special reference for microsatellite loci: G enetics 1996; 142: 1061-1064.

20 Excoffier L, Smouse PE, Q uattro J M : A nalysis of molecular variance inferred from metric distances among D NA haplotypes: application to human mitochondrial DNA restriction data. G enetics 1992; 131: 479-491. 